\title{
Nucleotide diversity in the mitochondrial and nuclear compartments of Chlamydomonas reinhardtii: investigating the origins of genome architecture David Roy Smith and Robert W Lee*
}

\author{
Address: Department of Biology, Dalhousie University, Halifax, Nova Scotia, Canada \\ Email: David Roy Smith - smithdr@dal.ca; Robert W Lee* - robert.lee@dal.ca \\ * Corresponding author
}

Published: 21 May 2008

BMC Evolutionary Biology 2008, 8:156 doi:10.1 |86/147|-2|48-8-156
Received: 4 March 2008

Accepted: 21 May 2008

This article is available from: http://www.biomedcentral.com/I47I-2/48/8/I56

(c) 2008 Smith and Lee; licensee BioMed Central Ltd.

This is an Open Access article distributed under the terms of the Creative Commons Attribution License (http://creativecommons.org/licenses/by/2.0), which permits unrestricted use, distribution, and reproduction in any medium, provided the original work is properly cited.

\begin{abstract}
Background: The magnitude of intronic and intergenic DNA can vary substantially both within and among evolutionary lineages; however, the forces responsible for this disparity in genome compactness are conjectural. One explanation, termed the mutational-burden hypothesis, posits that genome compactness is primarily driven by two nonadaptive processes: mutation and random genetic drift - the effects of which can be discerned by measuring the nucleotide diversity at silent sites $\left(\pi_{\text {silent }}\right)$, defined as noncoding sites and the synonymous sites of protein-coding regions. The mutational-burden hypothesis holds that $\pi_{\text {silent }}$ is negatively correlated to genome compactness. We used the model organism Chlamydomonas reinhardtii, which has a streamlined, coding-dense mitochondrial genome and an noncompact, intron-rich nuclear genome, to investigate the mutational-burden hypothesis. For measuring $\pi_{\text {silent }}$ we sequenced the complete mitochondrial genome and portions of 7 nuclear genes from 7 geographical isolates of $C$. reinhardtii.

Results: We found significantly more nucleotide diversity in the nuclear compartment of $C$. reinhardtii than in the mitochondrial compartment: net values of $\pi_{\text {silent }}$ for the nuclear and mitochondrial genomes were $32 \times 10^{-3}$ and $8.5 \times 10^{-3}$, respectively; and when insertions and deletions (indels) are factored in, these values become $49 \times 10^{-3}$ for the nuclear DNA and II $\times 10^{-}$ 3 for the mitochondrial DNA (mtDNA). Furthermore, our investigations of $C$. reinhardtii revealed 4 previously undiscovered mitochondrial introns, one of which contains a fragment of the largesubunit (LSU) rRNA gene and another of which is found in a region of the LSU-rRNA gene not previously reported (for any taxon) to contain introns.

Conclusion: At first glance our results are in opposition to the mutational-burden hypothesis: $\pi_{\text {silent }}$ was approximately 4 times greater in the nuclear compartment of $C$. reinhardtii relative to the mitochondrial compartment. However, when we consider the encumbrance of noncoding DNA in each of these $C$. reinhardtii compartments, we conclude that introns in the mtDNA impose a greater burden than those in the nuclear DNA and suggest that the same may be true for the intergenic regions. Overall, we cannot reject the mutational-burden hypothesis and feel that more data on nucleotide diversity from green algae and other protists are needed.
\end{abstract}




\section{Background}

Genomic sequence data from the three domains of life have revealed a prodigious range of genome compactnesses; however, our knowledge of the processes responsible for this gamut of genomic architectures is contentious. One explanation is the mutational-burden hypothesis [1-4], which posits that genome compactness (defined by the proportion of intronic and intergenic DNA) is primarily driven by nonadaptive processes: namely, mutation and random genetic drift. The mutational-burden hypothesis asserts that noncoding DNA (i.e., intronic and intergenic DNA) is a genetic liability because it is a target for deleterious and potentially lethal mutations, such as mutations affecting sequences involved with intron splicing and gene regulation. The hypothesis maintains that species with large effective population sizes $\left(N_{e}\right)$ are more efficient at purging, or preventing the proliferation of, hazardous-noncoding DNA because they experience less random genetic drift and thereby increase the efficacy of natural selection. The mutational-burden hypothesis holds that the product of $N_{e}$ and the mutation rate $(\mu)$ drives genome compactness; consequently, species whose genomes are coding rich should have a higher $N_{e} \mu$ than those whose genomes carry a surfeit of intronic and intergenic DNA.

Insights into $N_{e}$ and $\mu$ can be acquired by measuring the nucleotide diversity at silent sites $\left(\pi_{\text {silent }}\right)$, which are defined as noncoding sites and synonymous sites within protein-coding DNA. Since, for a diploid population at mutation-drift equilibrium, the rate at which new variation is introduced to a neutral-nucleotide site in two randomly compared alleles is equivalent to $2 \mu$ (twice the mutation rate), and the rate at which variation is lost from a neutral site is $1 / 2 N_{e^{\prime}}$ then the average number of nucleotide differences per neutral site is equivalent to the ratio of these two rates: $4 N_{e} \mu$. Because silent sites are typically regarded as among the most neutrally evolving positions in a genome, measures of $\pi_{\text {silent }}$ can provide an estimate of $4 N_{e} \mu$. This formula can be simplified by substituting $N_{g^{\prime}}$ the effective number of genes per locus in a population, for $N_{e^{\prime}}$ giving a final equation of $\pi_{\text {silent }}=2 N_{g} \mu$, where $N_{g}$ is equal to $N_{e}$ for nuclear genes of haploid species and about one-half $N_{e}$ for uniparentally-transmitted organelle genes [5]. Uniparentally-transmitted organelle genomes (mitochondrial or chloroplast) are generally considered haploid, despite being present in multiple copies per cell, because heteroplasmy (the existence of more than one organelle-genome haplotype in the same individual) is rare. Moreover, in this instance, $N_{g}$ is reduced further by the fact that during sexual reproduction only one of the parental sexual types transmits organelle genes to the next generation.
Large-scale studies have found a positive correlation between $\pi_{\text {silent }}$ and genome compactness: in the compact genomes of prokaryotes $\pi_{\text {silent }}$ tends to be $>50 \times 10^{-3}$; in the more bloated nuclear genomes of invertebrates and land plants it is in the range of $3 \times 10^{-3}$ to $15 \times 10^{-3}$; and in the nuclear genomes of vertebrates, where noncoding DNA predominates, it appears to lie between $2 \times 10^{-3}$ and $4 \times 10^{-3}[1]$. There is evidence that this trend is also found in organelle genomes: comparative studies of nucleotide diversity in mitochondrial DNA (mtDNA) indicate that in the diminutive, coding-dense mitochondrial genomes of mammals $\pi_{\text {silent }}$ is around $40 \times 10^{-3}$, whereas in the expanded mitochondrial genomes of land plants it is estimated to be $<0.4 \times 10^{-3}$ [3]. This disparity in $\pi_{\text {silent }}$ between land-plant and mammalian mitochondrial genomes is believed to be a reflection of the high mutation rates in mammalian mtDNA and the low mutation rates typically found in land-plant mtDNA. Mutation rates have also been invoked to explain why, despite similar proposed values of $N_{g^{\prime}}$ the mitochondrial and nuclear genomes of mammals have opposite coding densities - in mammals estimates of $\mu$ for mtDNA are roughly 30 times those for nuclear DNA [3]. Although the relationship between $\pi_{\text {silent }}$ and genome architecture is intriguing, the empirical data from which these correlations were derived are limited to a relatively small number of taxa and are generally skewed towards multicellular animals, with an overall lack of data for unicellular eukaryotes, especially green algae.

The model organism Chlamydomonas reinhardtii, a unicellular green alga of the chlorophycean class, is an excellent system for studying the evolution of genome compactness because it has a large, intron-rich nuclear genome and a small, compact mitochondrial genome, yet both genomes appear to have a similar mutation rate $[6,7]$. The nuclear genome of $C$. reinhardtii, which has been sequenced to 95\% completion, is approximately 121 megabases (Mb), with about $17 \%$ of the nucleotides coding for proteins and structural RNAs [8]. Furthermore, the genome has an abundance of introns ( $\sim 7$ per protein-coding gene), and the average intron length is longer than that of many eukaryotes and is more similar to multicellular organisms than to protists. In contrast, the mitochondrial genome of the standard laboratory strains of $C$. reinhardtii (derived from the Ebersold-Levine line) is streamlined, having a size of $15.8 \mathrm{~kb}$ and containing only 13 genes [9-11]. Moreover, at $82 \%$ coding it is one of the most compact mitochondrial genomes available from green algae (for a compilation see [12]), and although the mtDNA of one geographical isolate of $C$. reinhardtii (CC-1373) has an optional intron in $c o b$ [13] it is still > 75\% coding; this strain is often referred to as Chlamydomonas smithii but is in fact a member of the C. reinhardtii species [14]. 
According to the mutational-burden hypothesis, we would expect $C$. reinhardtii to have a high degree of silentsite nucleotide diversity in its mitochondrial genome (reflecting a large $2 N_{g} \mu$ ) and a low degree of silent-site nucleotide diversity in its nuclear genome (reflecting a small $2 N_{g} \mu$ ). To test this hypothesis and to investigate the correlation of $2 N_{g} \mu$ with genome compactness, we measured $\pi_{\text {silent }}$ in the mitochondrial and nuclear compartments from various geographical isolates of $C$. reinhardtii.

\section{Results}

\section{Strains and their genetic loci}

Seven geographical isolates of $C$. reinhardtii were employed in this study; their strain numbers, mating types, origins of isolation, and strain abbreviations are presented in Table 1. To access levels of genetic diversity we sequenced the complete mitochondrial genome and portions of 7 single-copy nuclear genes from each of the 7 isolates. A genetic map of the C. reinhardtii mitochondrial genome is shown in Figure 1, and partial genetic maps of the 7 nuclear genes are shown in Figure 2. We sequenced the entire mtDNA in order to employ both intergenic regions and synonymous sites in our calculations of $\pi_{\text {silent }}$ - previous studies on genetic diversity in mitochondrial genomes, due to a paucity of intraspecific sequence data, have tended to use only synonymous sites for estimating $\pi_{\text {silent }}$. Moreover, whole mtDNA sequences from C. reinhardtii allow for the comparison of synonymous-site nucleotide diversity $\left(\pi_{\text {syn }}\right)$ in the standard mitochondrial protein-coding genes to that of $r t l$, a mitochondrial open reading frame (ORF) in the $C$. reinhardtii mtDNA coding for a putative reverse-transcriptase-like protein [15]. It has been suggested that synonymous sites in $r t l$ are under less selective constraints than those of the standard mtDNA protein-coding genes and that they may be more appropriate for estimating the neutral mutation rate in the mitochondrial compartment [7]. For the nuclear loci, we sequenced mostly introns rather than exons because it is believed that in the C. reinhardtii nuclear genome intronic sites are more neutrally evolving than synonymous sites and may give more reliable estimates of the neutral mutation rate [6]. Sequences for two of the nuclear loci from the 7 isolates have been previously reported [16-18] allowing us to confirm both our strain assignations and sequencing methods.

We were able to obtain the complete mtDNA sequence from an 8th strain of C. reinhardtii (CC-503) by collecting and assembling mtDNA sequences that were generated from the $\mathrm{C}$. reinhardtii nuclear-genome sequencing project $[8,19]$. Both C. reinhardtii CC-503 and C. reinhardtii CC-277 (one of the 7 isolates described in Table 1) are cell-wall-less mutants recovered from the same "Ebersold-Levine" wild-type background of C. reinhardtii, but they have been separated for at least 35 years [20]. The mtDNA sequence of C. reinhardtii CC-503 is identical to that of C. reinhardtii CC-277; and when we downloaded the sequences of the 7 nuclear loci for $\mathrm{C}$. reinhardtii CC503, they too were identical to those of C. reinhardtii CC277. Therefore, for the purpose of this study we will be considering C. reinhardtii MA-1 as synonymous with C. reinhardtii CC-277 and CC-503.

Prior to this study a complete mtDNA sequence for $C$. reinhardtii was already available (Genbank accession number NC 001638); this sequence, which resulted from the accumulated efforts of multiple parties, mostly came from C. reinhardtii CC-277, or in some cases from strains having the same genetic background as $C$. reinhardtii CC277. The mtDNA sequence of $C$. reinhardtii CC-277 presented here differs at 46 positions relative to NC 001638; because 44 of these 46 differences are also present in the mtDNA of the other six C. reinhardtii isolates described here and because the $C$. reinhardtii CC-277 mtDNA sequence from this study was shown to be identical to the C. reinhardtii CC-503 mitochondrial genome, we feel that our version of the C. reinhardtii CC-277 mtDNA is currently the most accurate and that the discrepancies between our sequence and NC_001638 are the result of sequencing errors in the latter.

It is important to note that our annotation of the C. reinhardtii mitochondrial genome (Figure 1) does not contain the so-called rRNA-coding modules L2b and L3a. In previous studies each these modules was presumed to code for a non-core region of the large-subunit (LSU) rRNA

Table I: Chlamydomonas reinhardtii strains used in this study.

\begin{tabular}{llllll}
\hline Strain & Mating Type & Strain Synonym & Geographical Origin (USA) & Abbreviation a & Reference \\
\hline CC-277 & $m t^{+}$ & cwl5 & Amherst, Massachusetts & MA-I & Harris 1989 [20] \\
CC-1373 & $m t^{+}$ & C. smithii & South Deerfield, Massachusetts & MA-2 & Hoshaw and Ettl 1966 [4I] \\
CC-1952 & $m t^{-}$ & C. grossii & Plymouth, Minnesota & MN & Gross et al. 1988 [42] \\
CC-2342 & $m t^{-}$ & Jarvik 6 & Pittsburgh, Pennsylvania & PA-I & Spanier et al. 1992 [43] \\
CC-2344 & $m t^{+}$ & Jarvik 356 & Malvern, Pennsylvania & PA-2 & Spanier et al. 1992 [43] \\
CC-2931 & $m t^{-}$ & Harris 6 & Durham, North Carolina & NC & Harris 1989 [20] \\
CC-2343 & $m t^{+}$ & Jarvik I24 & Melbourne, Florida & FL & Spanier et al. 1992 [43] \\
\hline
\end{tabular}

a Strain abbreviations are based on the USA state from which the strains were isolated. 


\section{Chlamydomonas reinhardtii mitochondrial genome}

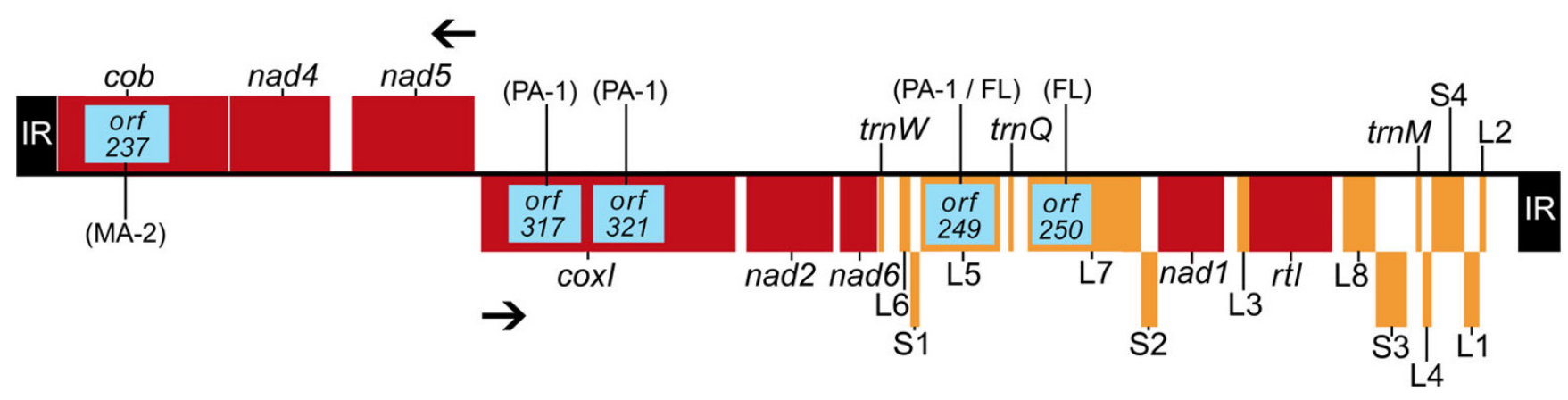

$1 \mathrm{~kb}$

Figure I

Genetic map of the Chlamydomonas reinhardtii mitochondrial genome, including all currently identified optional introns. Protein-coding regions and regions encoding structural RNAs are red and orange, respectively. SI-S4 represent the small-subunit rRNA-coding modules; LI-L8 represent the large-subunit rRNA-coding modules. The terminal inverted repeats (IR) are black. Intronic regions and their open reading frames are boxed in blue inside their associated genes. The $C$. reinhardtii strains (Table I) in which the different introns occur are labelled in parentheses. Solid arrows denote the transcriptional polarities. Note: due to the presence/absence of introns among the different strains, the size of the $C$. reinhardtii mitochondrial genome can vary from $15,782 \mathrm{nt}$ to $18,990 \mathrm{nt}$.

[21]. However, because sequence homologs of L2b and L3a have not been identified in the mtDNA of close relatives to $C$. reinhardtii $[7,22,23]$, or in any other genome, we have classified these regions as intergenic DNA, and have treated them as such for all genetic analyses.

\section{Nucleotide diversity}

Summary statistics of the nucleotide diversity in C. reinhardtii are shown in Table 2. Two measures of nucleotide diversity were used to calculate variation within the $C$. reinhardtii mitochondrial and nuclear genomes: $\pi$, which is the average number of pair-wise nucleotide differences per site between sequences in a sample [24], and $\theta_{\mathrm{W}^{\prime}}$ which is based on the number of polymorphic sites in a sample of sequences but is independent of their frequency [25]. With respect to both measures, the nuclear compartment shows significantly more silent-site nucleotide diversity than the mitochondrial compartment: net values of $\pi_{\text {silent }}$ for the nuclear DNA and mtDNA were $31.96 \times 10^{-}$ 3 and $8.54 \times 10^{-3}$, respectively. Net values of $\theta_{\mathrm{W}}$ at silent sites are slightly higher at $33.02 \times 10^{-3}$ for the nuclear compartment and $9.1810^{-3}$ for the mitochondrial compartment. In all cases, silent sites in the various nuclear loci show more diversity than the silent sites in the mitochondrial compartment. The only exception to this is the nuclear gene $C B L P$, which has less synonymous-site diversity $\left(\pi_{\text {syn }}=2.77 \times 10^{-3}\right)$ than that of the mitochondrial protein-coding regions. Within the mitochondrial compartment, diversity at intergenic and synonymous sites is similar $\left(8.92 \times 10^{-3}\right.$ vs. $\left.8.52 \times 10^{-3}\right)$, as is the diversity of protein-coding regions and regions coding for structural RNAs $\left(2.06 \times 10^{-3}\right.$ vs. $\left.2.42 \times 10^{-3}\right)$. The mitochondrial gene $r t l$, which encodes a putative reverse transcriptase, shows more diversity than the other mitochondrial protein-coding genes when all 3 codon sites are considered $\left(3.07 \times 10^{-3}\right.$ vs. $\left.2.06 \times 10^{-3}\right)$ and slightly less diversity when looking only at synonymous sites $\left(7.88 \times 10^{-3}\right.$ vs. $\left.8.52 \times 10^{-3}\right)$; however, it is unlikely that these observations are statistically significant.

\section{Insertions and deletions}

For both the nuclear and mitochondrial compartments, insertions and deletions (indels) represent a large proportion of the observed polymorphisms (Table 2). In our alignments of the nuclear loci from the 7 different strains of $C$. reinhardtii, $36 \%$ of mismatched nucleotides result from indels. The nuclear indels range from 1-31 nucleotides (nt) in length and have an average size of $4.5 \mathrm{nt}$. In the mitochondrial compartment indels represent $20 \%$ of the mismatched nucleotides. The mitochondrial indels range from 1-6 nt in length and have an average size of $2.5 \mathrm{nt}$. It is important to note that our estimates of nucleotide diversity shown in Table 2 are derived from sites in the alignment where all seven strains of $C$. reinhardtii have a nucleotide; therefore, sites corresponding to indels were removed from the alignment. If our methods for calculating $\pi$ are modified to include indels (by counting each gap in the alignment as a nucleotide change) the overall val- 
PDK

$970 \mathrm{nt}$

scaffold 3

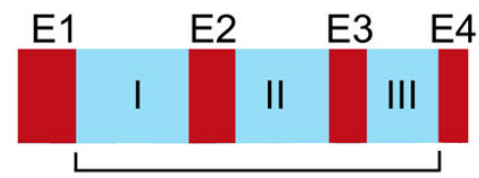

MAT3

$1060 \mathrm{nt}$

scaffold 10

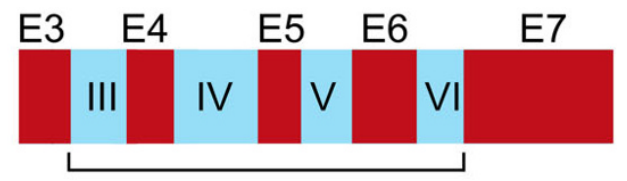

SFA

$1250 \mathrm{nt}$ scaffold 10

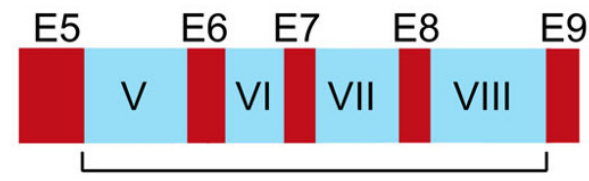

YPT4

$825 \mathrm{nt}$

scaffold 24

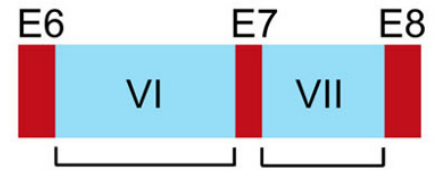

CBLP

$1100 \mathrm{nt}$

scaffold 38

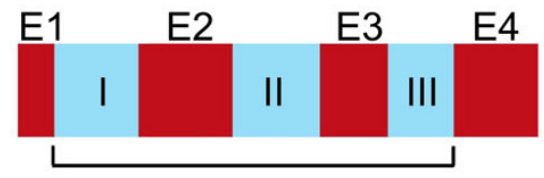

PETC

$960 \mathrm{nt}$ scaffold 41
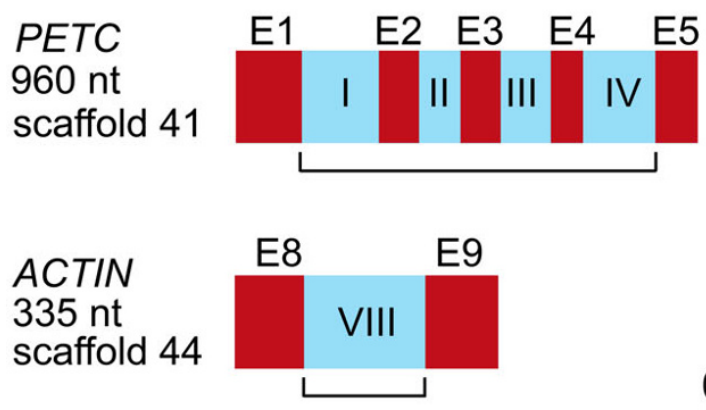

$\underline{0.25 \mathrm{~kb}}$

Figure 2

Partial genetic maps of the 7 Chlamydomonas reinhardtii nuclear-encoded genes employed in the analysis. The bracketed segment beneath each map represents the region that was PCR amplified. Left of each map is the name of the gene, the approximate size of the region that was PCR amplified, and the location of the gene within the $C$. reinhardtii nuclear genome - locations are based on the $C$. reinhardtii draft nuclear genome sequence version 3.0 [8]. Exons are red; they are labelled with an "E" and a number denoting their position within the gene. Introns are blue and are labelled with a roman numeral denoting their location within the gene. Note: each of these genes is present only once in the $C$. reinhardtii nuclear genome.

ues of $\pi_{\text {silent }}$ in the nuclear and mitochondrial compartments become $49.27 \times 10^{-3}\left( \pm 4.89 \times 10^{-3}\right)$ and $10.93 \times 10$ $3\left( \pm 1.96 \times 10^{-3}\right)$, respectively.

\section{Testing for neutrality}

Two statistical tests were performed on the mitochondrial and nuclear datasets to examine for traces of selection: Tajima's $D$-test, which compares the average number of nucleotide differences between pairs of sequences to the total number of segregating sites [24], and the McDonaldKreitman test, which compares the ratio of nonsynonymous to synonymous differences observed within a species to that observed between species [26]. Tajima's $D$ is slightly negative in all cases pertaining to the mitochondrial compartment and in most cases pertaining to the nuclear compartment, but it is slightly positive for a few of the nuclear loci (the exons of MAT3 and PDK, and the introns of CBLP, PETC, PDK, and ACTIN) (Table 2). In no case is Tajima's $D$-test statistically significant. The McDonald-Kreitman test was performed by comparing the ratio of nonsynonymous to synonymous polymorphisms within $C$. reinhardtii to the ratio of nonsynonymous to synonymous fixed differences between $C$. reinhardtii and Chlamydomonas incerta (one of the closest known noninterfertile relatives of C. reinhardtii [27]) (Table 3) - this was done for all of the protein-coding regions surveyed in this study. Overall, no significant departures from neutral expectations were detected for any of the mitochondrial or nuclear loci, and in no case is the McDonald-Kreitman test statistically significant.

\section{Mitochondrial introns}

Three of the C. reinhardtii strains (PA-1, MA-2, and FL) have introns in their mtDNA (Figure 1). C. reinhardtii MA2 has a single intron, inserted into $c o b$; $C$. reinhardtii FL has 2 introns, one in the L5-rRNA-coding module (the L5intron) and one in the L7-rRNA-coding module (the L7intron); and C. reinhardtii PA-2 has 3 introns, two in cox 1 and the L5-intron (note: the DNA sequence of the L5intron in C. reinhardtii PA-2 is identical to that of C. reinhardtii FL). Of these introns only that of $c o b$ in C. reinhardtii MA-2 has been previously described [13]. Like the intron of $c o b$ in C. reinhardtii MA-2, each of the 4 introns presented here has an ORF for which the deduced amino acid sequence shows similarity to a LAGLIDADG-type endonuclease. RT-PCR experiments confirm that all five introns, including their ORFs, are spliced-out in mature transcripts. Secondary-structure modelling suggests that the two introns in cox1 are group I introns belonging to subgroup D. Our analyses of the L5- and L7-introns suggest that they lack the core sequence and potential secondary structure necessary to be classified as either group I or group II introns; thus, at the present time they are considered highly-degenerate "unclassified" introns. A 35-nt duplicated portion of the L5-rRNA-coding module is found within the 5' end of the L5-intron; RT-PCR experiments validate that this segment is in fact a component of the intron. The insertion sites of the L5- and L7-introns within the $C$. reinhardtii mtDNA and the nature of the 
Table 2: Nucleotide diversity in the mitochondrial and nuclear compartments of Chlamydomonas reinhardtii.

\begin{tabular}{|c|c|c|c|c|c|c|c|c|c|c|}
\hline & & & \# of sites & $S$ & $\begin{array}{r}\text { \# of Indelsg } \\
\text { (length nt) }\end{array}$ & $\begin{array}{r}\pi \times 10^{-3} \\
\left(\mathrm{SD} \times 10^{-3}\right)\end{array}$ & $\begin{array}{r}\theta_{W} \times 10^{-3} \\
\left(S D \times 10^{-3}\right.\end{array}$ & $\pi_{\text {syn }} \times 10^{-3}$ & $\pi_{\mathrm{ns}} \times 10^{-3}$ & $\begin{array}{c}\text { Tajima's } D \text { test } \\
\text { (P value) }\end{array}$ \\
\hline \multirow[t]{6}{*}{ mtDNA } & \multicolumn{2}{|l|}{ Complete genome $\mathrm{e}^{\mathrm{a}}$} & 15280 & 134 & $15(36)$ & $3.35(0.68)$ & $3.66(0.31)$ & -- & -- & $-0.49(>0.10)$ \\
\hline & \multicolumn{2}{|l|}{ Protein-coding $\mathrm{b}$} & 8154 & 44 & I (6) & $2.06(0.43)$ & $2.20(0.33)$ & 8.52 & 0 & $-0.38(>0.10)$ \\
\hline & \multicolumn{2}{|c|}{ structural RNA genes ${ }^{c}$} & 3502 & 23 & $4(4)$ & $2.42(0.42)$ & $2.68(0.56)$ & --- & --- & $-0.55(>0.10)$ \\
\hline & \multicolumn{2}{|l|}{$r t l$} & 1118 & 9 & $1(3)$ & $3.07(0.87)$ & $3.29(1.10)$ & 7.88 & 1.89 & $-0.35(>0.10)$ \\
\hline & \multicolumn{2}{|l|}{ Intergenic ${ }^{d}$} & 2434 & 58 & $9(23)$ & $8.92(1.88)$ & $9.73(1.28)$ & --- & --- & $-0.48(>0.10)$ \\
\hline & \multicolumn{2}{|l|}{ Silent sitese } & 5152 & 99 & $12(26)$ & $8.54(1.03)$ & $9.18(0.65)$ & --- & --- & --- \\
\hline \multirow{15}{*}{$\begin{array}{l}\text { Nuclear } \\
\text { DNA }\end{array}$} & \multicolumn{2}{|l|}{ Intronic (overall) } & 4294 & 359 & $47(216)$ & $33.50(3.15)$ & $33.81(1.79)$ & -- & --- & $-0.16(>0.10)$ \\
\hline & \multicolumn{2}{|l|}{ Silent sites ${ }^{f}$} & 4824 & 381 & $50(219)$ & $31.96(3.03)$ & $33.02(1.88)$ & --- & --- & --- \\
\hline & \multicolumn{2}{|l|}{ Exonic (overall) } & 1614 & 25 & I (9) & $6.02(0.99)$ & $6.58(1.29)$ & 19.57 & 1.42 & $-0.48(>0.10)$ \\
\hline & \multirow[t]{5}{*}{ Exonic (by gene) } & $C B L P$ & 420 & I & 0 & $0.68(0.47)$ & $0.97(0.97)$ & 2.77 & 0 & $-1.00(>0.10)$ \\
\hline & & PETC & 300 & 2 & 0 & $2.54(0.78)$ & $2.72(1.92)$ & 9.69 & 0 & $-0.27(>0.10)$ \\
\hline & & PDK & 222 & 3 & 0 & $6.86(1.88)$ & $5.52(3.18)$ & 26.35 & 0 & $1.10(>0.10)$ \\
\hline & & MAT3 & 408 & 10 & I (9) & $10.50(2.70)$ & 10.00 & 24.37 & 4.77 & $0.27(>0.10)$ \\
\hline & & SFA & 264 & 9 & 0 & $9.74(2.80)$ & $13.91(4.64)$ & 41.14 & 0 & $-1.59(>0.10)$ \\
\hline & \multirow[t]{7}{*}{ Intronic (by gene) } & $C B L P$ & 578 & 77 & $8(75)$ & $58.30(7.41)$ & $54.30(6.20)$ & --- & --- & $0.26(>0.10)$ \\
\hline & & PETC & 621 & 43 & $3(5)$ & $30.21(6.07)$ & $28.26(4.31)$ & --- & --- & $0.39(>0.10)$ \\
\hline & & PDK & 691 & 37 & $8(42)$ & $23.22(5.58)$ & 21.86 & --- & --- & $0.20(>0.10)$ \\
\hline & & МАT3 & 560 & 30 & $4(25)$ & $21.60(3.03)$ & $21.87(3.99)$ & --- & --- & $-0.07(>0.10)$ \\
\hline & & SFA & 847 & 98 & $12(15)$ & $43.57(4.53)$ & 48.67 (4.77) & --- & --- & $-0.61(>0.10)$ \\
\hline & & YPT4 & 790 & 47 & $9(39)$ & $22.66(4.50)$ & 24.28 & --- & --- & $-0.38(>0.10)$ \\
\hline & & ACTIN & 207 & 27 & $3(15)$ & $53.37(8.18)$ & $53.37(10.2)$ & --- & --- & $0.01(>0.10)$ \\
\hline
\end{tabular}

Note--S, number of segregating sites; Indels, insertion and deletions; $\pi$, nucleotide diversity; $\theta_{W}$, Theta (per-site) from Watterson estimator; $\pi_{\text {syn }}$, nucleotide diversity at synonymous sites; $\pi_{\mathrm{n}}$, nucleotide diversity at nonsynonymous sites; $\mathrm{SD}$, standard deviation.

a Includes only I telomere.

$\mathrm{b}$ Includes all protein-coding genes excluding rtl.

c Includes rRNA- and tRNA-coding regions.

d Includes intergenic regions and I telomere.

e Includes synonymous sites, intergenic regions, and I telomere.

$\mathrm{f}$ Includes synonymous sites and intronic regions.

$\mathrm{g}$ Nucleotide diversity does not include indels. Consecutive indel sites are counted as single event. Indel length refers to the sum of all indels.

repeat found within the $\mathrm{L} 5$-intron are described in Figure 3A, B, and 3C, respectively. The insertion sites of the L5and L7-introns in context to the C. reinhardtii LSU rRNA sequence are shown in Figure 3D.

\section{Discussion}

\section{Accounting for the differences in $\pi_{\text {silent }}$}

Before we interpret our data on nucleotide diversity in relation to the mutational-burden hypothesis, let us first try to account for the values of $\pi_{\text {silent }}$ that we observed. Overall, in C. reinhardtii we found 3.7-fold more nucleotide diversity at silent sites in the nuclear compartment than in the mitochondrial compartment; and when indels are taken into consideration, $\pi_{\text {silent }}$ appears to be 4.5 times greater in the nuclear DNA compared to the mtDNA. Assuming that $\pi_{\text {silent }}$ is equal to $2 N_{g} \mu$, we can discuss our findings on nucleotide diversity in relation to $\mu$ and $N_{g}$.

In a recent study that compared silent-site substitution rates in the mitochondrial and nuclear genomes between C. reinhardtii and $C$. incerta, it was concluded that the mutation rate in the nuclear compartment of these taxa is approximately the same as that in the mitochondrial compartment $[6,7]$. If is similar in both the nuclear DNA and mtDNA of $C$. reinhardtii, then it appears that differences in $N_{g}$ would have to explain the disparity in nucleotide diversity that we observe between these genomes.
In order to arrive at the values of $\pi_{\text {silent }}$ observed in this study, $N_{g}$ would have to be higher for the nuclear genome than for the mitochondrial genome (again, assuming equal mutation rates); there are a few reasons why this might be the case. For the haploid alga $C$. reinhardtii, nuclear genes are inherited biparentally and mitochondrial genes are inherited uniparentally [28]. As mentioned earlier, this is thought to reduce $N_{g}$ in the mitochondrial compartment by approximately one-half relative to that in the nucleus [5]. Thus, for $C$. reinhardtii we might expect a value of $\pi_{\text {silent }}$ in the mitochondrial compartment to be around one-half of what is observed in the nuclear compartment. Uniparental inheritance also implies that the mtDNA of $C$. reinhardtii has less opportunity for recombination than the nuclear DNA, which may make the mitochondrial genome as a whole more susceptible to the effects of selective sweeps and purifying selection, both of which can reduce $N_{g^{\prime}}$ resulting in an even smaller than expected value of $\pi_{\text {silent }}$ in the mitochondrial compartment $[29,30]$. One way to detect the influences of selection is with the McDonald-Kreitman test, where positive selection is inferred if the test returns a value for the neutrality index $(\mathrm{NI})<1$, and purifying selection is indicated by NI > 1 [29-31]. Although the results of the MacDonaldKreitman test for C. reinhardtii versus $C$. incerta showed no significant departure from neutral expectations, values of NI were $<1$ for $r t$ and the concatenated sequence of the 
Table 3: McDonald-Kreitman test comparing the ratio of nonsynonymous to synonymous differences within Chlamydomonas reinhardtii to that found between $C$. reinhardtii and Chlamydomonas incerta.

\begin{tabular}{|c|c|c|c|c|c|c|c|}
\hline & & & $\begin{array}{l}\text { Polymorphisms within } C . \\
\text { reinhardtii }\end{array}$ & $\begin{array}{l}\text { Substitutions between } C \text {. } \\
\text { reinhardtii and } C \text {. incerta }\end{array}$ & $\mathrm{NI}$ & $G$ & $P$ \\
\hline \multirow[t]{4}{*}{ mtDNA } & Protein-codinga & Nonsynonymous & 3 & 61 & 0.653 & 0.533 & 0.465 \\
\hline & & Synonymous & 36 & 478 & & & \\
\hline & $r t l$ & Nonsynonymous & 5 & 111 & 0.746 & 0.185 & 0.667 \\
\hline & & Synonymous & 4 & 119 & & & \\
\hline \multirow[t]{12}{*}{ Nuclear DNA } & Exonic (overall)b & Nonsynonymous & 1 & 18 & 0.159 & 4.837 & 0.027 \\
\hline & & Synonymous & 21 & 60 & & & \\
\hline & $C B L P$ & Nonsynonymous & 0 & 0 & undef & undef & undef \\
\hline & & Synonymous & 1 & I & & & \\
\hline & PETC & Nonsynonymous & 0 & 3 & 0.000 & undef & undef \\
\hline & & Synonymous & 2 & 4 & & & \\
\hline & PDK & Nonsynonymous & 0 & 2 & 0.000 & undef & undef \\
\hline & & Synonymous & 3 & 18 & & & \\
\hline & MAT3 & Nonsynonymous & 1 & 9 & 0.677 & 1.081 & 0.298 \\
\hline & & Synonymous & 6 & 18 & & & \\
\hline & SFA & Nonsynonymous & 0 & 4 & 0.000 & undef & undef \\
\hline & & Synonymous & 9 & 19 & & & \\
\hline
\end{tabular}

Note--NI, neutrality index (ratio of nonsynonymous to synonymous polymorphisms within C. reinhardtii compared to the ratio of nonsynonymous to synonymous fixed differences between $C$. reinhardtii and $C$. incerta); $G$, G-test of independence (determines if the proportion of nonsynonymous substitutions is independent of whether the substitutions are fixed or polymorphic); $P$, probability of $G$-test; undef, undefined. $C$. incerta data came from [6] and [7]. Note: in no case was the McDonald-Kreitman test statistically significant.

a Includes all protein coding genes excluding rtl.

b Concatenated exons from all 5 nuclear genes.

standard mitochondrial protein-coding regions (Table 3), which might be an indication of positive selection. Moreover, Tajima's $D$ test returned negative values for all of the mtDNA regions that were examined (Table 2) - negative values of Tajima's $D$ test can be an indication of a recent selective sweep of a linked mutation [32] - but again these findings were not statistically significant.

A further consideration is that the mutation rate in the $C$. reinhardtii mitochondrial compartment may be slightly lower than that in the nuclear compartment. When Popescu and Lee [7] estimated $\mu$ to be similar for both the nuclear and mitochondrial genomes of $C$. reinhardtii, they used the synonymous substitution rate in $r t l$ (which was about double that of the standard mitochondrial proteincoding regions) as an estimate of the neutral mutation rate in the mitochondrial compartment. In contrast, we found $\pi_{\text {syn }}$ in $r t l$ to be similar to that of the standard mitochondrial protein-coding regions $\left(7.88 \times 10^{-3}\right.$ vs. $8.52 \times$ $10^{-3}$ ), and although this could be an artefact of a small sample size, it might suggest that Popescu and Lee overestimated $\mu$ in the C. reinhardtii mtDNA.

\section{$\Pi_{\text {silent }}$ in relation to previous studies on $\mathrm{C}$. reinhardtii and other unicellular eukaryotes}

Our estimates of $\pi_{\text {silent }}$ in the nuclear and mitochondrial genomes of $C$. reinhardtii are approximately $32 \times 10^{-3}$ and $8.5 \times 10^{-3}$, respectively; and with indels factored in, these values become $\sim 50 \times 10^{-3}$ for the nuclear DNA and about
$11 \times 10^{-3}$ for the mtDNA. The only other estimates of nucleotide diversity in the C. reinhardtii nuclear and mitochondrial genomes that we could find were that of Lynch and Connery [1], who, using $11 \mathrm{~kb}$ of mostly noncoding nuclear DNA from C. reinhardtii strains MA-1 and MN, found $\pi_{\text {silent }}$ in the nuclear compartment to be $\sim 40 \times 10^{-3}$ (they estimated $N_{e} \mu$ to be $\sim 20 \times 10^{-3}$ ), which is in agreement with our observations. With respect to other green algae, we are unaware of any reported estimates of withinpopulation silent-site variation in either nuclear or mitochondrial genomes.

There is an overall lack of data on $\pi_{\text {silent }}$ for unicellular species; however, the data that are available are relatively consistent with our results for $C$. reinhardtii. Among unicellular fungi, average values of $\pi_{\text {silent }}$ in the nuclear and mitochondrial compartments (based on data for 3 different species) were estimated to be $50 \times 10^{-3}$ and $12 \times$ $10^{-3}$, respectively $[1,3]$, which is comparable to what we observed in C. reinhardtii. Furthermore, the genera Paramecium and Trypanosoma appear to have ratios of $\pi_{\mathrm{si}-}$ lent(mitochondrion) $/ \pi_{\text {silent(nucleus) }}$ of approximately 0.4 [3], which is within the range of our estimates for C. reinhardtii (0.2-0.25). All studies deriving measures of $\pi_{\text {silent }}$ from the mitochondrial genome of unicellular eukaryotes have used 3 or fewer loci for their estimates and have tended to focus only on synonymous sites, which makes our analysis one of the most comprehensive for any mitochondrial genome from a unicellular eukaryote to date. 


\section{A Intron insertion sites within the mtDNA}

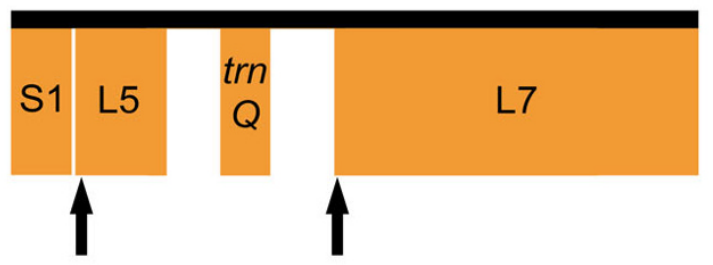

\section{B Intron of the L5-rRNA-coding module}

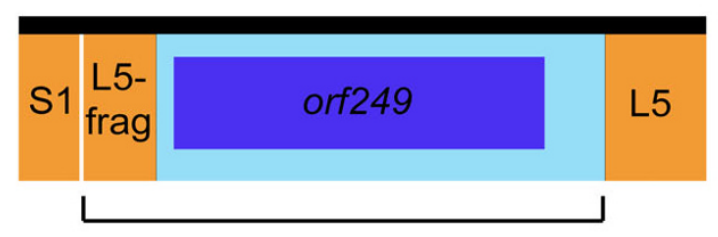

\section{Intron of the L7-rRNA-coding module}

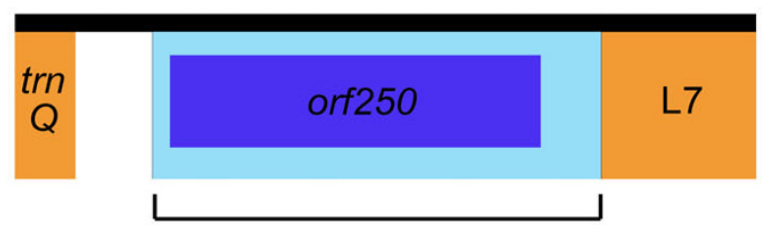

D Intron insertion sites within the LSU rRNA
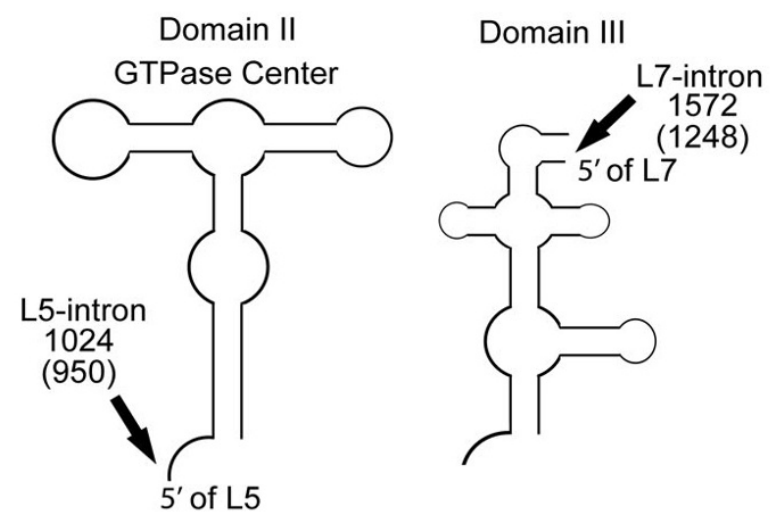

Figure 3

Schema of the introns in the L5- and L7-rRNA-coding modules. The vertical arrows in $\mathbf{A}$ show the intron insertion sites within the $C$. reinhardtii mtDNA. B and C depict the introns in the L5- and L7-rRNA-coding modules, respectively; rRNA-coding regions are orange; introns are light blue; intronic open reading frames are boxed in dark blue within their respective introns; L5-frag refers to a duplicated segment of the L5-rRNAcoding module (the first $35 \mathrm{nt}$ of the module are duplicated); bracketed portions of the map represent regions that were shown to be spliced-out in mature transcripts. $\mathbf{D}$ depicts the intron insertion sites in the context of the large subunit (LSU) ribosomal RNA sequence of $C$. reinhardtii; arrows point to the region where the introns are inserted; numbers above the arrows denote the position of the residue that immediately precedes the insertion site: un-bracketed numbers correspond to the residue in the $23 \mathrm{~S}$ rRNA gene of Escherichia coli [44] and bracketed numbers correspond to the residue in the LSU-rRNA secondary-structure model of Boer and Gray [2I]. Note: the $C$. reinhardtii strains in which these introns occur are shown in Figure $\mathrm{I}$.
It is also of interest to compare the nucleotide diversity of C. reinhardtii to that of multicellular species. Our estimation of nucleotide diversity in the nuclear genome of $C$. reinhardtii is much greater than that observed for animals: $\sim 9$ times larger than the average estimate for mammals $\left(3.6 \times 10^{-3}\right)$, and 2 times greater than the average for invertebrates, which is believed to be $<14.8 \times 10^{-3}$ [1]. Our approximation of $\pi_{\text {silent }}$ for the mitochondrial genome of C. reinhardtii is much smaller than that of animals: 0.2 times the average for mammals $\left(40 \times 10^{-3}\right)$ and $0.1-0.6$ times the average for invertebrates $\left(11 \times 10^{-3}-67 \times 10^{-3}\right)$ [2]. In comparison to land plants, C. reinhardtii has twice the amount of silent-site nucleotide variation in its nuclear genome ( $\pi_{\text {silent }}$ in land plants is estimated to be $\sim 15 \times 10-3$ ), and although we are unaware of any reliable assessments of $\pi_{\text {silent }}$ in land plant mtDNA, it is purported to be $<0.4 \times 10^{-3}[3]$, which is less than 0.05 times what we observed for the $C$. reinhardtii mtDNA.

\section{Testing the mutational-burden hypothesis}

At first glance, our estimates of nucleotide diversity in the mitochondrial and nuclear genomes of C. reinhardtii appear contrary to what would be expected under the mutational-burden hypothesis. We found $\pi_{\text {silent }}$ to be $\sim 4$ times greater in the nuclear compartment than in the mitochondrial compartment, but based on the streamlined nature of the C. reinhardtii mitochondrial genome in relation to its noncompact nuclear genome, one might expect the mutational-burden hypothesis to predict a greater value of $\pi_{\text {silent }}$ for the mtDNA. However, before we conclude that our findings are in opposition to the mutation-burden hypothesis, we must first consider what the actual "encumbrance" of noncoding DNA is for the mitochondrial and nuclear genomes in C. reinhardtii.

As described earlier, the basic premise of the mutationburden hypothesis is that noncoding DNA magnifies the target site for deleterious mutations, thereby, increasing the susceptibility of a genome to degenerative changes. The mutational disadvantage of noncoding DNA, therefore, is critically dependent on: 1) the number of nucleotides that are associated with gene function $(n)$, and 2 ) the per-nucleotide mutation rate $(\mu)$. These two terms can be combined to define the overall mutational disadvantage $(s)$, where $s=n \mu[3,33]$. It is predicted that the threshold in a genome below which noncoding DNA can proliferate is $2 N_{g} s<1$, or alternatively $2 N_{g} \mu<1 / n[3,33]$. Although it is difficult to estimate $n$ for intergenic regions, values of $n$ for intronic regions can be predicted with reasonable confidence. For the spliceosomal introns of eukaryotic genomes, $n$ is believed to be around 25 [33], which gives a threshold for intron colonization in the nuclear compartment of $2 \mathrm{~N}_{g} \mu<0.04$. Because mitochondrial introns are self-splicing and do not rely on a spliceosome for excision they have more nucleotides that are 
critical for proper splicing; thus, we can conservatively say that for mitochondrial introns $n$ is between 75-100 (see [34] for a review on mitochondrial-intron folding), giving a threshold for intron proliferation in the mitochondrial compartment of $\sim 2 N_{g} \mu<0.01$. Based on the mutationalburden hypothesis, our estimates of $2 N_{g} \mu$ (i.e. $\pi_{\text {silent }}$ ) in the nuclear and mitochondrial compartments of $C$. reinhardtii, whether including or excluding the influence of indels, lie too close to the predicted thresholds for intron proliferation in these genomes to accurately forecast intron abundance.

We do not know what the encumbrance of intergenic regions is for either the nuclear or the mitochondrial genome of C. reinhardtii, but if it is (or was at some point in the past) substantially higher for the mtDNA than the nuclear DNA, it would indicate that the mitochondrial compartment is a less permissive environment for the proliferation of intergenic DNA. And although this is highly speculative, there is one reason why this might be the case. In the $C$. reinhardtii mitochondrial genome, mature-RNA transcripts are generated by precise endonucleolytic cleavage of long polycistronic precursor-messenger RNAs; it is believed that these immature transcripts are cleaved in regions of the RNA corresponding to intergenic sites in the mitochondrial genome, and that processing is critically dependent on the primary sequence and the secondary structure of these regions [9]. This implies that the intergenic sites in the C. reinhardtii mitochondrial genome may have a large mutational burden associated with them, perhaps large enough to impose a barrier on the amplitude of intergenic mtDNA. Moreover, the polycistronic nature of the C. reinhardtii mitochondrial transcripts suggest that the regulatory elements within the intergenic mtDNA carry the increased burden of being responsible for many genes - a burden not typically associated with the monocystronic gene regulation of nuclear DNA [35].

If the burden of intronic and intergenic DNA is higher in the mtDNA of green algae and other protists, then we might expect to find very low values of $\pi_{\text {silent }}$ in the mitochondrial genomes of species from these groups that have an abundance of intronic and intergenic sequences - for examples see references $[12,36,37]$.

It is worth noting that the $C$. reinhardtii chloroplast genome when compared to its mitochondrial counterpart has a similarly low density of introns but a substantially greater proportion of intergenic DNA [38]. Using $1500 \mathrm{nt}$ of chloroplast DNA (composed of the petA gene and a single intergenic region) we found no nucleotide polymorphisms in any of the 7 geographical isolates of $C$. reinhardtii employed in this study (our unpublished data). For a genome rich in intergenic DNA this value is consist- ent with what one might expect under the mutationalburden hypothesis.

\section{Novel mitochondrial introns}

An unforeseen consequence of this study is the discovery of 4 previously unreported $C$. reinhardtii mitochondrial introns, two of which (the L5- and L7-introns) are unusual. Although a detailed description of these introns is beyond the scope of this paper, they each contain a characteristic that is notable: 1) the L5-intron carries a $35 \mathrm{nt}$ portion of the L5-rRNA-coding module within the 5 -end of its DNA sequence - this is an unprecedented feature for a mitochondrial intron. And 2) the insertion site of the L7-intron in relation to the C. reinhardtii LSU-rRNA secondary-structure model of Boer and Gray [21] corresponds to domain III (Figure 3); we believe this to be the first example (for any taxon) of an intron found in domain III of the LSU rRNA.

The discovery of 4 new optional introns in the C. reinhardtii mtDNA does not alter the notion that in C. reinhardtii the mitochondrial compartment is significantly more compact than the nuclear compartment: the mitochondrial genome of $C$. reinhardtii PA-1, the isolate with the most introns, is still $~ 67 \%$ coding compared to $<20 \%$ for the nuclear genome.

\section{Conclusion}

The main objective of this study was to investigate genome compactness from a population-genetic perspective and, in doing so, test a contemporary hypothesis regarding the origins of genome architecture - the mutational-burden hypothesis. Our findings may not appear to be in full agreement with the mutational-burden hypothesis, we found $\sim 4$ times more nucleotide diversity in the nuclear compartment of $C$. reinhardtii relative to the mitochondrial compartment. However, when the $2 N_{g} \mu$-threshold for the proliferation of intronic and intergenic DNA is considered, we conclude that introns impose a greater burden on $C$. reinhardtii mtDNA and suggest that the intergenic regions of this genome do so as well. Overall, we cannot reject the mutational-burden hypothesis.

\section{Methods}

Strains, culture conditions, and DNA and RNA extractions All of the C. reinhardtii strains employed in this study were obtained from the Chlamydomonas Center at Duke University in July of 2006, with the exception to C. reinhardtii CC-277, which was obtained from the same source in 1991. Clonal cultures of each strain were prepared from a single vegetative colony recovered on agar medium [20]. For each of the seven strains, total genomic DNA was extracted using the DNeasy Plant Mini Kit (Qiagen, Germantown, MD), and total RNA was extracted using the RNeasy Plant Mini Kit (Qiagen). 


\section{Strain confirmation}

To verify that the 7 C. reinhardtii isolates had been assigned the correct strain numbers we compared our sequences of the ypt4-VI, ypt4-VII, and act-VIII introns for each isolate to those obtained in other studies [16-18] from the corresponding isolates. The results confirmed that the $7 \mathrm{C}$. reinhardtii isolates employed here are the same as those used in previous reports.

\section{Amplification and sequencing of genetic loci}

A PCR-based approach was used to amplify the mtDNA and the nuclear loci examined in this study. PCR experiments were performed in High Fidelity Platinum SuperMix (Invitrogen, Carlsbad, CA) using total genomic DNA as the template. Reverse-transcriptase (RT) PCR reactions were performed with the SuperScript III One-Step RT-PCR System (Invitrogen) following the manufacturer's protocol. PCR and RT-PCR products were purified using the QIAquick PCR Purification Kit (Qiagen). The purified products were sequenced on both strands at the Macrogen sequencing facility, Rockville, MD, USA.

\section{C. reinhardtii strain $\mathrm{CC}-503$}

The complete mitochondrial genome of $C$. reinhardtii strain CC-503 (cw92 $m t^{+}$) was obtained by collecting and assembling mtDNA sequences generated from the C. reinhardtii nuclear genome sequencing project [19]. These sequences were acquired by blasting the complete $C$. reinhardtii mitochondrial genome against the following databases at the United States Department of Energy Joint Genome Institute (DOE JGI): C. reinhardtii v3.1-unplaced reads and $C$. reinhardtii v3.1-bonus scaffolds. Blast hits showing > 99\% similarity to $C$. reinhardtii mtDNA were downloaded and assembled. All mitochondrial hits were subsequently checked against the draft nuclear genome of C. reinhardtii (v3.0 unmasked assembly) to insure that no nuclear mitochondrial DNA sequences (NUMTs) were collected. Our assembly of the downloaded mtDNA sequences contains over 500 chromatogram reads and gives a complete $C$. reinhardtii $\mathrm{CC}-503$ mitochondrial genome with 15-fold coverage.

\section{Sequence analyses}

To ensure that the 7 nuclear-encoded genes employed in this study are present only once in the $C$. reinhardtii nuclear genome we blasted each of the 7 sequences against the C. reinhardtii draft nuclear genome [19]. All 7 genes returned a single hit, which is consistent with the hypothesis that these genes are present in single copies in the $C$. reinhardtii nuclear genome. The mitochondrial sequences obtained from each of the seven strains were also blasted against the C. reinhardtii draft nuclear genome to confirm that they are not NUMTs: the blast results suggest that there are very few copies of mitochondrial sequences in the nuclear compartment and the few that are present are highly degenerate; therefore, we are confident that none of the mtDNA sequences presented in this study are NUMTs. The mitochondrial introns of $\operatorname{cox} 1$ and their secondary structures were identified using RNAweasel [34]. DnaSP 4.0 [39] was used for calculating all measures of genetic diversity. Nucleotide diversity and its standard deviation were calculated using equation 10.6 and 10.3 of Nei [40], respectively. Theta was calculated using equation 3 of Tajima [32]. The MacDonald-Kreitman test [26] and Tajima's D test [24] were performed in DnaSP.

\section{Accession numbers}

Nucleotide-sequence accession numbers for the sequences that were employed in this study are shown in Table 4.

\section{Authors' contributions}

DRS carried out the molecular studies, data analyses, and drafted the manuscript. RWL helped in interpreting the data and revising the manuscript. Both DRS and RWL have read and approved the final version of this manuscript.

Table 4: Genbank accession numbers of the Chlamydomonas reinhardtii sequences employed in this study.

\begin{tabular}{|c|c|c|c|c|c|c|c|c|c|}
\hline Strain & mtDNAa $^{a}$ & CBLPa & PETCa & $P D K^{\mathrm{a}}$ & MAT3a & $S F A^{a}$ & ACTIN-VIIIb & YPT4-VIb & YPT4-VIIb \\
\hline CC-277c & EU306622 & EU306630 & EU30665I & EU306644 & EU306632 & EU306658 & D50838 & U13167 & U13167 \\
\hline CC- 1373 & EU306617 & EU306625 & EU306646 & EU306639 & EU306633 & EU306653 & U7057I & U559II & U55912 \\
\hline CC-1952 & EU30662I & EU306626 & EU306647 & EU306640 & EU306634 & EU306654 & U70563 & U55893 & U55894 \\
\hline CC-2342 & EU306620 & EU306627 & EU306648 & EU30664I & EU306635 & EU306655 & U70569 & U55905 & U55906 \\
\hline CC-2343 & EU306623 & EU306628 & EU306649 & EU306642 & EU306636 & EU306656 & U7056I & U55889 & U55890 \\
\hline CC-2344 & EU306619 & EU306629 & EU306650 & EU306643 & EU306637 & EU306657 & U70562 & U5589I & U55892 \\
\hline CC-293I & EU306618 & EU306624 & EU306645 & EU306638 & EU30663I & EU306652 & U70568 & U5590I & U55902 \\
\hline
\end{tabular}

a Present study.

b Sequences from [18] except for D50838, which is from [17], and UI3167, which is from [16].

c The mitochondrial genome from this strain was shown to be identical to that of $C$. reinhardtii CC-503. 


\section{Acknowledgements}

We thankfully acknowledge Joseph Bielawski and Joel Malek for helpful comments as well as Sean Farmer and Tudor Borza who carried out preliminary analyses at the inception of this study. This work was supported by a grant to RWL from the Natural Sciences and Engineering Research Council (NSERC) of Canada. DRS received a Canada Graduate Scholarship from NSERC.

\section{References}

I. Lynch M, Conery JS: The origins of genome complexity. Science 2003, 302: | $401-1404$.

2. Lynch M: Streamlining and simplification of microbial genome architecture. Annu Rev Microbiol 2006, 60:327-349.

3. Lynch M, Koskella B, Schaack S: Mutation pressure and the evolution of organelle genomic architecture. Science 2006, 3II:1727-1730.

4. Lynch M: The Origins of Genome Architecture Sunderland: Sinauer Associates, Inc; 2007.

5. Birky $\mathrm{CW} J \mathrm{~J}$, Fuerst $\mathrm{P}$, Maryama $\mathrm{T}$ : Organelle gene diversity under migration, mutation, and drift: equilibrium expectations, approach to equilibrium, effect of heteroplasmic cells, and comparison to nuclear genes. Genetics 1989, 121:613-627.

6. Popescu CE, Borza T, Bielawski JP, Lee RW: Evolutionary rates and expression level in Chlamydomonas. Genetics 2006, 172:1567-1576.

7. Popescu CE, Lee RW: Mitochondrial genome sequence evolution in Chlamydomonas. Genetics 2007, 175:819-826.

8. Merchant SS, Prochnik SE, Vallon O, (II7 co-authors), et al.: The Chlamydomonas genome reveals the evolution of key animal and plant functions. Science 2007, 3 1 8:245-250.

9. Gray MW, Boer PH: Organization and expression of algal (Chlamydomonas reinhardtii) mitochondrial DNA. Philos Trans $R$ Soc Lond B Biol Sci 1988, 31 19:135-147.

10. Michaelis G, Vahrenholz C, Pratje E: Mitochondrial DNA of Chlamydomonas reinhardtii: the gene for apocytochrome $b$ and the complete functional map of the $15.8 \mathrm{~kb}$ DNA. Mol Gen Genet 1990, 223:211-216.

II. Vahrenholz C, Riemen G, Pratje E, Dujon B, Michaelis G: Mitochondrial DNA of Chlamydomonas reinhardtii: the structure of the ends of the linear 15.8-kb genome suggests mechanisms for DNA replication. Curr Genet 1993, 24:241-247.

12. Pombert JF, Beauchamp P, Otis C, Lemieux C, Turmel M: The complete mitochondrial DNA sequence of the green alga Oltmannsiellopsis viridis: evolutionary trends of the mitochondrial genome in the Ulvophyceae. Curr Genet 2006 , 50:137-147.

13. Colleaux L, Michel-Wolwertz MR, Matagne RF, Dujon B: The apocytochrome $b$ gene of Chlamydomonas smithii contains a mobile intron related to both Saccharomyces and Neurospora introns. Mol Gen Genet 1990, 223:288-296.

14. Boynton JE, Harris EH, Burkhart BD, Lamerson PM, Gillham NW: Transmission of mitochondrial and chloroplast genomes in crosses of Chlamydomonas. Proc Natl Acad Sci USA 1987, 84:239|-2395.

15. Boer PH, Gray MW: Genes encoding a subunit of respiratory NADH dehydrogenase (NDI) and a reverse transcriptaselike protein (RTL) are linked to ribosomal RNA pieces in Chlamydomonas reinhardtii mitochondrial DNA. EMBO J 1988 , 7:350I-3508.

16. Dietmaier W, Fabry S, Huber H, Schmitt R: Analysis of a family of ypt genes and their products from Chlamydomonas reinhardtii. Gene 1995, 158:41-50.

17. Sugase $Y$, Hirono M, Kindle KL, Kamiya R: Cloning and characterization of the actin-encoding gene of Chlamydomonas reinhardtii. Gene 1995, 168:|17-121.

18. Liss M, Kirk DL, Beyser K, Fabry S: Intron sequences provide a tool for high-resolution phylogenetic analysis of volvocine algae. Curr Genet 1997, 31:2 14-227.

19. JGI Chlamydomonas reinhardtii v3.0 [http://genome.jgi-psf.org/ Chlre3/Chlre3.home.html]
20. Harris EH: The Chlamydomonas Sourcebook San Diego: Academic Press; 1989.

21. Boer PH, Gray MW: Scrambled ribosomal RNA gene pieces in Chlamydomonas reinhardtii mitochondrial DNA. Cell 1988 55:399-4II

22. Denovan-Wright EM, Lee RW: Comparative structure and genomic organization of the discontinuous mitochondrial ribosomal RNA genes of Chlamydomonas eugametos and Chlamydomonas reinhardtii. J Mol Biol I994, 24 I :298-3 I I.

23. Fan J, Schnare MN, Lee RW: Characterization of fragmented mitochondrial ribosomal RNAs of the colorless green alga Polytomella parva. Nucleic Acids Res 2003, 31:769-778.

24. Tajima F: Statistical method for testing the neutral mutation hypothesis by DNA polymorphism. Genetics 1989, I 23:585-595.

25. Watterson GA: On the number of segregating sites in genetical models without recombination. Theor Pop Biol 1975, 7:256-276.

26. McDonald $\mathrm{J}$, Kreitman M: Adaptive protein evolution at the Adh locus in Drosophila. Nature 1991, 35 1:652-654.

27. Pröschold T, Harris EH, Coleman A: Portrait of a species: Chlamydomonas reinhardtii. Genetics 2005, 170:1601-1610.

28. Harris EH: Chlamydomonas as a model organism. Annu Rev Plant Physiol Plant Mol Biol 200I, 52:160-174.

29. Bazin E, Glémin S, Galtier N: Population size does not influence mitochondrial genetic diversity in animals. Science 2006 , 3 | 2:570-572.

30. Meiklejohn CD, Montooth KL, Rand DM: Positive and negative selection on the mitochondrial genome. Trends Genet 2007 23:259-263.

3I. Rand DM, Kann LM: Mutation and selection at silent and replacement sites in the evolution of animal mitochondrial DNA. Genetica 1998, 103:393-407.

32. Tajima F: Measurement of DNA polymorphisms. In Mechanisms of Molecular Evolution Edited by: Takahat N and Clark AG. Sunderland: Sinauer Associates, Inc; 1993:37-59.

33. Lynch M: Intron evolution as a population-genetic process. Proc Natl Acad Sci USA 2002, 99:6I I 8-6123.

34. Lang BF, Laforest MJ, Burger G: Mitochondrial introns: a critical view. Trends Genet 2007, 23:1 19-125.

35. Gurley WB, O'Grady K, Czarnecka-Verner E, Lawit SI: General transcription factors and the core promoter: ancient roots. In Regulation of Transcription in Plants Edited by: Grasser KD. Oxford: Blackwell Publishing, Ltd; 2006: I-2I.

36. Turmel $M$, Otis $C$, Lemieux $C$ : An unexpectedly large and loosely packed mitochondrial genome in the charophycean green alga Chlorokybus atmophyticus. BMC Genomics 2007, 8: 137 .

37. Forget L, Ustinova J, Wang Z, Huss VA, Lang BF: Hyaloraphidium curvatum: a linear mitochondrial genome, tRNA editing, and an evolutionary link to lower fungi. Mol Biol Evol 2002, 19:310-319.

38. Maul JE, Lilly JW, Cui L, dePamphilis CW, Miller W, Harris EH, Stern DB: The Chlamydomonas reinhardtii plastid chromosome: islands of genes in a sea of repeats. Plant Cell 2002, 14:2659-2679.

39. Rozas J, Sánchez-DelBarrio JC, Messeguer X, Rozas R: DnaSP, DNA polymorphism analyses by the coalescent and other methods. Bioinformatics 2003, 19:2496-2497.

40. Nei M: Molecular Evolutionary Genetics New York: Columbia University Press; 1987

4l. Hoshaw RW, Ettl H: Chlamydomonas smithii sp. nov.: a chlamydomonad interfertile with Chlamydomonas reinhardtii. J Phycol 1966, 2:93-96.

42. Gross $\mathrm{CH}$, Ranum LPW, Lefebvre PA: Extensive restriction fragment length polymorphisms in a new isolate of Chlamydomonas reinhardtii. Curr Genet 1988, 13:503-508.

43. Spanier JG, Graham JE, Jarvik JW: Isolation and preliminary characterization of three Chlaymydomonas strains interfertile with Chlamydomonas reinhardtii (Chlorophyta). J Phycol 1992, 28:822-828

44. Cannone JJ, Subramanian S, Schnare MN, Collett JR, D'Souza LM, Du Y, Feng B, Lin N, Madabusi LV, Müller KM, Pande N, Shang Z, Yu N, Gutell RR: The comparative RNA web (CRW) site: an online database of comparative sequence and structure information for ribosomal, intron, and other RNAs. BMC Bioinformatics 2002, 3: 15 . 